\title{
The Hypoglycemic Effects of Soy Isoflavones on Postmenopausal Women
}

\author{
SHAO-YI CHENG, M.D., M.P.H., ${ }^{1}$ NING-SING SHAW, Ph.D., ${ }^{3}$ \\ KE-SUNG TSAI, M.D., Ph.D., ${ }^{2}$ and CHING-YU CHEN, M.D. ${ }^{1}$
}

\begin{abstract}
Objectives: Soy isoflavones have many effects similar to those of estrogen and have become popular among postmenopausal women as an alternative for hormone replacement therapy (HRT). The purpose of this study was to determine the effects of soy isoflavones on glucose, insulin, and lipid profiles in postmenopausal Taiwanese women.

Methods: We conducted a randomized, double-blind, active placebo-controlled clinical trial to compare the effects of isoflavones with estrogen replacement therapy (ERT) on blood glucose, insulin, and lipid profiles in postmenopausal Taiwanese women. Thirty postmenopausal Taiwanese women were randomly assigned to two groups, and each received treatment for 6 months. The isoflavone group received $100 \mathrm{mg}$ isoflavone soft capsules, $300 \mathrm{mg}$ calcium, and a blank vitamin capsule per day. The estrogen active control group received $0.625 \mathrm{mg}$ conjugated estrogen, $300 \mathrm{mg}$ calcium, and blank isoflavone soft capsules per day. We measured baseline levels of fasting blood sugar, insulin, lipid profiles, and isoflavone concentrations prior to the study and repeated the same measurements every 3 months for a total duration of 6 months.

Results: Two-way ANOVA revealed that fasting glucose $(p<0.001)$ and insulin $(p<0.005)$ levels were significantly affected by estrogen and isoflavone treatments after 6 months. For the isoflavone group, the average blood genistein concentration was 6-10 times higher than those of the estrogen group. Within the same periods, the fasting blood glucose was reduced to $83 \%$ and $85 \%$ of the baseline levels, and insulin was reduced to $56 \%$ and $67 \%$ of the baseline levels, respectively, in the estrogen and isoflavone groups.

Conclusions: Soy isoflavones (100 $\mathrm{mg}$ ) and $0.625 \mathrm{mg}$ conjugated estrogen equally lower fasting blood glucose and insulin levels in postmenopausal women.
\end{abstract}

\section{INTRODUCTION}

$\mathbf{P}$ Hytoestrogens are naturally occurRing substances and are classified into isoflavones, coumestans, and lignans. Isoflavones are chemically and structurally similar to estradiol $\left(\mathrm{E}_{2}\right)$, but the physiological effect is $10^{2}-10^{5}$ less than that of $\mathrm{E}_{2} \cdot{ }^{1}$ Small-scale studies have demonstrated that isoflavones can relieve menopausal symptoms, lower total cholesterol (TCHO) and low-density lipoprotein (LDL), prevent osteoporosis, and concomitantly lower the rate of high-risk mammo-

\footnotetext{
${ }^{1}$ Department of Family Medicine and ${ }^{2}$ Department of Laboratory Medicine, National Taiwan University Hospital, Taipei, Taiwan.

${ }^{3}$ Institute of Microbiology and Biochemistry, National Taiwan University, Taipei, Taiwan.

This work was sponsored by National Taiwan University Hospital and Multipower Enterprise Corp.
} 
graphic parenchymal patterns. ${ }^{1-4}$ Among the various effects of isoflavones, the role of LDL reduction seems to be well documented; however, other metabolic effects, such as blood glucose control, are rarely investigated.

As unopposed estrogen can improve insulin resistance and hyperinsulinemia, ${ }^{5}$ we hypothesize that isoflavones can cause the same effect.

\section{MATERIALS AND METHODS}

\section{Participants}

Thirty postmenopausal women participated in the study through the department of family medicine outpatient clinic in April 2002. The inclusion criteria were aged 48-75-years; postmenopausal without menstrual cycle for at least 1 year, which was documented by serum $E_{2}$, follicle-stimulating hormone $(\mathrm{FSH})$, and luteinizing hormone $(\mathrm{LH})$ levels; body mass index (BMI) $>18.5 \mathrm{~kg} / \mathrm{m}^{2}$ and $<30 \mathrm{~kg} / \mathrm{m}^{2} ; \mathrm{Hb} \mathrm{A} 1 \mathrm{C}<10 \%$; and willing to comply with the protocol and sign the written informed consent. The exclusion criteria were vegetarian; undiagnosed vaginal bleeding; significant or pathological endometrial hyperplasia; IUD user; neurological diseases; known cardiovascular, cerebrovascular, or peripheral vascular disorders; uncontrolled hypertension with blood pressure >180/100 mm Hg; uncontrolled hypothyroidism; any renal disease with serum creatinine $>1.5 \mathrm{mg} / \mathrm{dl}$; abnormal liver function, with serum alanine aminotransferase (SALT) and serum alanine aminotransaminase (SAST) value $>2$-fold upper limits; mentally retarded; alcoholic; smoker; on hypoglycemic agents; on lipidlowering agent or taking hormone replacement treatment (HRT) or SERM or phytoestrogens in the 3 months before the study; and past history of malignancy.

\section{Study design}

Our study was a randomized, double-blind, active placebo-controlled human clinical trial. It lasted for 6 months. All participants were randomly assigned to one of two groups. The randomization procedure was based on a random number table that had an allocation ratio and was performed by the study assistant, who was also blinded. The isoflavone group was given $25-\mathrm{mg}$ isoflavone soft capsules (4 pills per day), a blank vitamin capsule, and $300 \mathrm{mg}$ calcium per day. The estrogen active control group was given blank isoflavone soft capsules ( 4 pills per day), $0.625 \mathrm{mg}$ conjugated equine estrogen (CEE), and $300 \mathrm{mg}$ calcium per day. Fasting blood samples were drawn from all the participants before initiation of the study, and baseline levels of glucose, insulin, TCHO, LDL cholesterol (LDL-C), triglycerides (TG), high-density lipoprotein cholesterol (HDL-C), and genistein were measured.

During the study, all subjects visited the outpatient clinic and received their monthly quota of medications on schedule every month. Body height and weight were measured at each visit. Blood samples were collected every 3 months, and measurements of glucose, insulin, TG, TCHO, LDL-C, HDL-C, and genistein were repeated. Subjects were advised not to eat food rich in isoflavones, such as soybean. They were instructed to report any problems and side effects associated with the medication. All subjects provided written informed consent.

\section{Supplements}

Isoflavone soft capsules with the ingredients originally from Novasoy (Archer Daniels Midland, Decatur, IL) were provided by Multipower Enterprise Corp. (Taipei, Taiwan). Each capsule contained evening primrose oil, daidzein, and genistein ( $\mathrm{w} / \mathrm{w}$ ratio 1:1:3) in conjugated form, with an equivalent of $25 \mathrm{mg}$ isoflavones. The genistein and daidzein content was verified using high-performance liquid chromatography (HPLC) according to the method of Thomas et al., ${ }^{6}$ with some modifications. All capsules were stored at ambient temperature after manufacture. The estrogen we used was $0.625 \mathrm{mg} \mathrm{CEE}$ (Premarin; Sheng-Da Pharmaceutical, Taipei, Taiwan), manufactured in Taiwan.

\section{Blood analyses}

Serum TG, TCHO, and LDL-C measurements were performed in the department of laboratory medicine at National Taiwan University Hospital, using enzymatic assays and Vitros 950 Chemistry Analyzer (Johnson \& Johnson, New Brunswick, NJ). Serum HDL-C was calculated according to the formula:

$$
\text { HDL }(\mathrm{mmol} / \mathrm{L})=\mathrm{TCHO}-\left(\frac{\mathrm{TG}}{2.2}\right)-\mathrm{LDL}-\mathrm{C}
$$

Serum glucose and insulin measurements were performed in Nan Chang Medical Laboratory 
Center (Taipei, Taiwan), using commercial reagents and the Hitachi 7070 automatic analyzer and Roche-ELESCYS 2010 for enzymatic assay and immunoassay, respectively.

Plasma genistein was extracted and quantified using HPLC according to the method of Thomas et al., ${ }^{6}$ with some modifications.

\section{Statistical analysis}

Descriptive data are presented as means \pm standard deviation (SD). Statistical analysis was performed with PC SAS for Windows software (version 8.2, Cary, NC). The difference in baseline characteristics was assessed using Student's $t$ test. The main effects of treatment and time on lipid profile and glucose, insulin, and genistein concentrations were assessed by two-way ANOVA. Differences among treatment and time groups were assessed by Duncan's multiple range test after controlling the baseline glucose and LDL-C levels using forward multiple regression analysis. A $p$ value $<0.05$ was considered statistically significant.

\section{RESULTS}

\section{Participants}

There were 30 subjects enrolled in the study and 28, completed the study, $17(94 \%)$ in the isoflavone group and $11(91 \%)$ in the estrogen group. One subject withdrew from the study because of abdominal pain, and one subject discontinued because of vaginal bleeding. The baseline characteristics are shown in Table 1. The subjects in the isoflavone group were slightly older, shorter, and lighter than those in the estrogen replacement group, although the differences were not significant. The fluctuation of BMI in both groups was also not significant during the study period.

\section{Blood genistein}

The baseline level of blood genistein averaged 0.48 and $0.46 \mu \mathrm{mol} / \mathrm{L}$ for the estogen and the isoflavone groups, respectively, and there was no significant difference between the two groups. Treatment significantly affected the blood genistein concentration $(p=0.031)$, and the isoflavone group had significantly higher levels than the estrogen group after 3 and 6 months of intervention (Table 2). For the estrogen group, the average blood genistein concentration decreased to $0.11 \mu \mathrm{mol} / \mathrm{L}$ after 3 months and to $0.07 \mu \mathrm{mol} / \mathrm{L}$ at 6 months. For the isoflavone group, the average blood genistein concentration increased to $0.74 \mu \mathrm{mol} / \mathrm{L}$ after 3 months and to $0.85 \mu \mathrm{mol} / \mathrm{L}$ at 6 months.

\section{Glucose, insulin, and lipid profiles}

For the estrogen group, the average glucose concentration was $118 \pm 46 \mathrm{mg} / \mathrm{dl}$ at baseline, which decreased markedly to $94 \pm 20 \mathrm{mg} / \mathrm{dl}$ after 3 months and to $92 \pm 21 \mathrm{mg} / \mathrm{dl}$ at 6 months. For the isoflavone group, the average glucose concentration was $102 \pm 18 \mathrm{mg} / \mathrm{dl}$ at baseline, which decreased to $90 \pm 24 \mathrm{mg} / \mathrm{dl}$ at 3 months and markedly to $81 \pm 25 \mathrm{mg} / \mathrm{dl}$ at 6 months. The duration of treatment significantly affected the glucose concentration $(p=0.001)$ in both groups. For the estrogen group, the insulin level averaged $11 \pm 9 \mu \mathrm{U} / \mathrm{ml}$ at baseline and decreased to $6 \pm 7$ and $6 \pm 11 \mu \mathrm{U} / \mathrm{ml}$ at 3 and 6 months, respectively. For the isoflavone group, the insulin level was $12 \pm 12 \mu \mathrm{U} / \mathrm{ml}$ at baseline, which decreased significantly to $4 \pm 3 \mu \mathrm{U} / \mathrm{ml}$ at 3 months and to $5 \pm 8 \mu \mathrm{U} / \mathrm{ml}$ at 6 months. The duration of treatment significantly affected the insulin levels $(p=$

Table 1. Baseline Characteristics of Postmenopausal Women Given EITHER ESTROGEN OR ISOFLAVONES FOR 6 MONTHS $^{\mathrm{a}}$

\begin{tabular}{lcc}
\hline Treatment group & $\begin{array}{c}\text { Estrogen group } \\
(\mathrm{n}=11)\end{array}$ & $\begin{array}{c}\text { Soy isoflavone group } \\
(\mathrm{n}=17)\end{array}$ \\
\hline Age, years & $58.1 \pm 6.0$ & $60.9 \pm 8.9$ \\
Height, meters & $1.57 \pm 0.05$ & $1.54 \pm 0.06$ \\
Weight, kilograms & $57.3 \pm 5.7$ & $52.1 \pm 6.5$ \\
Body mass index, $\mathrm{kg} / \mathrm{m}^{2}$ & $23.2 \pm 2.0$ & $21.8 \pm 2.6$ \\
LMP, years ${ }^{b}$ & $11.2 \pm 8.2$ & $16.1 \pm 11.4$ \\
\hline
\end{tabular}

aThe two groups were not significantly different as assessed by Student's $t$ test.

${ }^{\mathrm{b}} \mathrm{LMP}$, last menstrual period. 
Table 2. Effects of Estrogen and Soy Isoflavone Supplementation on Blood Genistein, Insulin, and Glucose Concentrations in Postmenopausal Women ${ }^{\mathrm{a}, \mathrm{b}}$

\begin{tabular}{|c|c|c|c|c|c|c|c|c|}
\hline \multirow{3}{*}{$\begin{array}{l}\text { Blood } \\
\text { parameters }\end{array}$} & \multicolumn{6}{|c|}{ Treatment groups and duration } & \multirow{2}{*}{\multicolumn{2}{|c|}{$\begin{array}{c}\mathrm{p} \text { values from } \\
\text { two-way } \\
\text { ANOVA }\end{array}$}} \\
\hline & \multicolumn{3}{|c|}{ Estrogen group $(\mathrm{n}=11)$} & \multicolumn{3}{|c|}{ Soy isoflavone group $(\mathrm{n}=17)$} & & \\
\hline & 0 months & 3 months & 6 months & 0 months & 3 months & 6 months & Time & Treatment \\
\hline $\begin{array}{l}\text { Genistein, } \\
\mu \text { mole/L }\end{array}$ & $0.48 \pm 0.76^{\mathrm{a}, \mathrm{b}}$ & $0.11 \pm 0.25^{b}$ & $0.07 \pm 0.22^{b}$ & $0.46 \pm 0.94^{\mathrm{a}, \mathrm{b}}$ & $0.74 \pm 0.65^{\mathrm{a}}$ & $0.85 \pm 0.81^{\mathrm{a}}$ & 0.942 & 0.031 \\
\hline Glucose, mg/dl & $118 \pm 46^{\mathrm{a}}$ & $94 \pm 20^{b}$ & $92 \pm 21^{b}$ & $102 \pm 18^{a, b}$ & $90 \pm 24^{b}$ & $81 \pm 25^{b}$ & 0.001 & 0.154 \\
\hline Insulin, $\mu \mathrm{IU} / \mathrm{ml}$ & $11 \pm 9^{a}$ & $6 \pm 7^{a, b}$ & $6 \pm 11^{\mathrm{a}, \mathrm{b}}$ & $12 \pm 12^{\mathrm{a}}$ & $4 \pm 3^{b}$ & $5 \pm 8^{\mathrm{a}, \mathrm{b}}$ & 0.005 & 0.940 \\
\hline
\end{tabular}

${ }^{\mathrm{a}, \mathrm{b}}$ Different superscript letters in each row indicate significant difference by Duncan's multiple range test, $p<0.05$.

'There was no significant interaction between time and treatment for all three parameters as tested by two-way ANOVA, $p<0.05$.

0.005) in both groups. Both blood glucose and insulin levels shifted toward lower values after the intervention with either estrogen or soy isoflavone (Fig. 1).

In contrast, the lipid profiles, including TG, TCHO, HDL-C, TCHO/HDL-C ratio, and LDL$\mathrm{C} / \mathrm{HDL}-\mathrm{C}$ ratio, in both groups were not significantly changed by the intervention (Table 3), except that soy isoflavone-treated subjects showed a significant increase in LDL-C at 6 months.

\section{DISCUSSION}

Cardiovascular disease (CVD) is the third leading cause of death for Taiwanese women. Apart from the close association with hypertension and hyperlipidemia, the rapidly increasing prevalence of diabetes is believed to be responsible. This has drawn the attention of both the medical field and the field of public health.

Compared to the west, acceptance of HRT is relatively low in Taiwanese women. Ever since

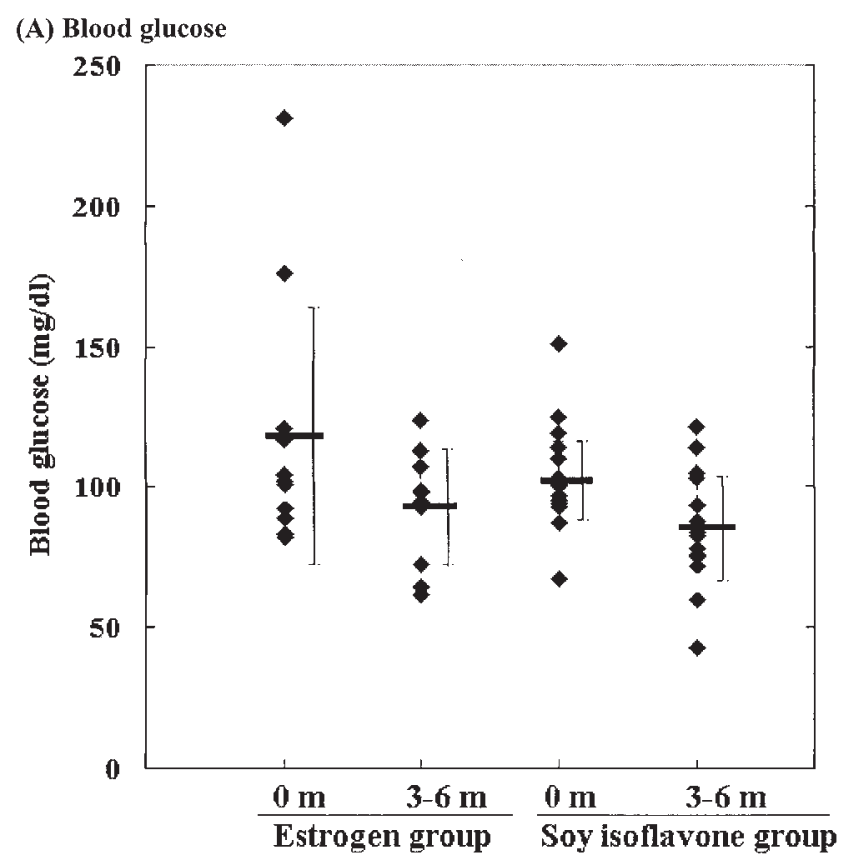

(B) Serum insulin

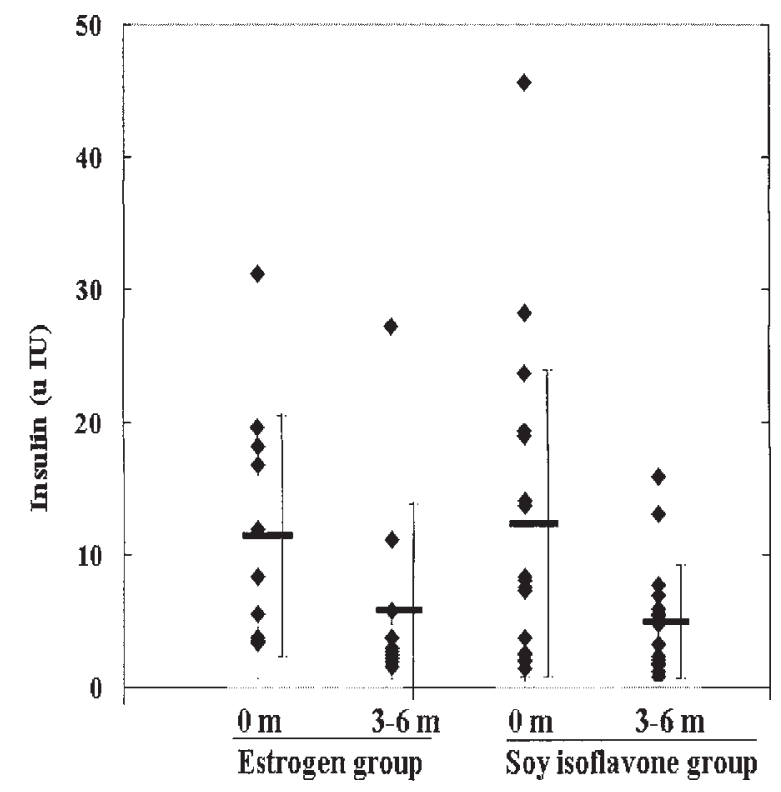

FIG. 1. Distribution pattern of blood glucose concentrations (A) and insulin levels (B) in postmenopausal women before and after estrogen or soy isoflavone supplementation for 6 months. Horizontal bar indicates group mean, and vertical line indicates 1 SD for the specified group. 
Table 3. Effects of Estrogen and Isoflavone Supplementation on Blood Lipid Profiles in Postmenopausal Women

\begin{tabular}{|c|c|c|c|c|c|c|}
\hline \multirow{3}{*}{$\begin{array}{l}\text { Blood } \\
\text { lipid profiles }\end{array}$} & \multicolumn{6}{|c|}{ Treatment groups and duration ${ }^{\mathrm{a}}$} \\
\hline & \multicolumn{3}{|c|}{ Estrogen group $(\mathrm{n}=11)$} & \multicolumn{3}{|c|}{ Soy isoflavone group $(\mathrm{n}=17)$} \\
\hline & 0 months & 3 months & 6 months & 0 months & 3 months & 6 months \\
\hline Triglycerides, mmole/L & $1.38 \pm 0.59$ & $1.66 \pm 0.84$ & $1.57 \pm 1.21$ & $1.26 \pm 0.49$ & $1.44 \pm 0.83$ & $1.28 \pm 0.55$ \\
\hline $\mathrm{TCHO}, \mathrm{mmole} / \mathrm{L}$ & $5.56 \pm 0.71$ & $5.85 \pm 0.99$ & $5.69 \pm 0.95$ & $5.11 \pm 0.83$ & $5.50 \pm 0.85$ & $5.38 \pm 0.84$ \\
\hline LDL-C, mmole/L $\mathrm{L}^{\mathrm{b}, \mathrm{c}, \mathrm{d}}$ & $3.39 \pm 0.75^{\mathrm{b}, \mathrm{c}}$ & $3.40 \pm 0.75^{\mathrm{b}, \mathrm{c}}$ & $3.87 \pm 0.89^{b}$ & $2.75 \pm 0.78^{d}$ & $3.23 \pm 0.62^{\mathrm{c}, \mathrm{d}}$ & $3.48 \pm 0.69^{b, c}$ \\
\hline HDL-C, mmole/L & $1.56 \pm 0.37$ & $1.71 \pm 0.47$ & $1.69 \pm 0.44$ & $1.84 \pm 0.76$ & $1.68 \pm 0.59$ & $1.70 \pm 0.52$ \\
\hline TCHO/HDL-C & $3.7 \pm 0.8$ & $3.6 \pm 1.0$ & $3.5 \pm 0.9$ & $3.1 \pm 1.1$ & $3.6 \pm 1.1$ & $3.3 \pm 0.8$ \\
\hline LDL/HDL-C & $2.3 \pm 0.7$ & $2.1 \pm 0.7$ & $2.4 \pm 0.8$ & $1.8 \pm 0.9$ & $2.1 \pm 0.8$ & $2.2 \pm 0.7$ \\
\hline
\end{tabular}

aThe main effects of treatment and duration on lipid profiles were not statistically significant as tested by two-way ANOVA, $p<0.05$.

$\mathrm{b}, \mathrm{c}, \mathrm{d}$ Different superscript letters in this row indicate significant difference by Duncan's multiple range test, $p<0.05$.

the report by the Women's Health Initiative $(\mathrm{WHI}),{ }^{7}$ the prevalence rate of taking HRT is even lower; many Taiwanese women discontinued HRT and turned to alternative medicine. Among the various treatments, phytoestrogens is one of the most popular.

The majority of studies on isoflavones have concentrated on their effects on blood lipids. Studies that have shown decreases in serum LDL$\mathrm{C}$ with soy proteins in humans generally have involved hypercholesterolemic subjects and inclusion of soy proteins in their treatment. Such an effect may be absent in a healthy population using purified isoflavones. ${ }^{8-14}$ In our study, dietary advice was kept to a minimum to avoid any major changes in the subjects' habitual diets. The results were consistent with recent findings using phytoestrogens, which found that isoflavone supplement alone had no effect on lipid profiles during the 6-month intervention.

Recent studies have provided evidence that phytoestrogens may be beneficial in the management of diabetes. In vitro studies have shown isoflavones to have antidiabetic properties, such as inhibiting intestinal brush border uptake of glucose, having $\alpha$-glucosidase inhibitor action, demonstrating tyrosine kinase inhibitory properties and multiple action on insulin release from pancreatic islet cells, and acting as peroxisome proliferator-activated receptor (PPAR- $\alpha$ and PPAR- $\gamma$ ) agonists. ${ }^{15-19}$ Human studies are few, but results are promising. Diets containing soy protein rich in isoflavones can reduce insulin levels in healthy postmenopausal women. Soy consumption also alleviates insulin resistance and impaired glycemic control in postmenopausal women with type 2 diabetes. ${ }^{20}$

In our study, after 6 months of intervention, the average blood genistein concentration for the isoflavone group was 10 times higher compared with those of the estrogen group. Within the same period, the fasting blood glucose level was reduced to $83 \%$ and $85 \%$ of the baseline levels and the insulin level was reduced to $56 \%$ and $67 \%$ of the baseline levels, respectively, in the estrogen and isoflavone groups. The effects of both estrogen and genistein treatment observed in our study were greater than the $16 \%$ reduction in fasting insulin after 3 years of HRT in the Postmenopausal Estrogen/Progestogen Intervention (PEPI) trial. ${ }^{21}$ This shows that isoflavones are as effective as estrogen in improving insulin resistance and glucose tolerance.

Two prior studies have reported phytoestrogen effects on glycemic control and insulin sensitivity in postmenopausal women. Jayagopal et al. ${ }^{22}$ demonstrated that dietary supplementation with $30 \mathrm{~g}$ soy proteins containing $132 \mathrm{mg}$ phytoestrogen for 12 weeks reduced fasting insulin by $8.09 \%$ in postmenopausal women with diet-controlled type 2 diabetes. Goodman-Gruen and Kritz-Silverstein ${ }^{23}$ examined the effects of usual, unsupplemented dietary isoflavones ranging from none to $13.9 \mathrm{mg} /$ day, with an average of $1.3 \pm 2.4$ $\mathrm{mg} /$ day, and observed a significant $20 \%$ reduction in fasting insulin in women consuming moderate to high isoflavones in their usual diet. In accordance with these studies, our results also support the insulin-reducing and hypoglycemic effects of soy isoflavones. These effects remained 
significant after controlling the baseline LDL-C levels by forward multiple regression analysis in our study. The results in our study and in the study of Goodman-Gruen and Kritz-Silverstein ${ }^{23}$ are similar in that insulin and glucose reduction occurred in the absence of any change in lipid profiles, including TCHO, HDL-C, TCHO/HDL$\mathrm{C}$ ratio, and LDL-C/HDL-C ratio, thus demonstrating a differential effect of soy isoflavones on blood lipid and glycemic controls in postmenopausal women.

\section{CONCLUSIONS}

As the dietary habits of Taiwanese women become more and more westernized, the increasing prevalence of obesity, hyperlipidemia, and hyperglycemia leads to a greater incidence of CVD. The accumulating evidence regarding the ability of phytoestrogens, especially isoflavones, to lower plasma lipid and blood glucose levels seems to provide postmenopausal women as alternative to HRT for potential cardioprotection. It also can foster a reappreciation of the benefits of the traditional diet.

\section{ACKNOWLEDGMENT}

We thank Mr. Hans Tu and Ms. Cindy Cheng for technical assistance.

\section{REFERENCES}

1. Glazier MG, Bowman MA. A review of the evidence for the use of phytoestrogens as a replacement for traditional estrogen replacement therapy. Arch Intern Med 2001;161:1161.

2. Jakes RW, Duffy SW, Ng FC, et al. Mammographic parenchymal patterns and self-reported soy intake in Singapore Chinese women. Cancer Epidemiol Biomarkers Prev 2002;11:608.

3. Potter MS, Baum JA, Teng H, Stillman RJ, Shay NF, Erdman JW Jr. Soy protein and isoflavones: Their effects on blood lipids and bone density in postmenopausal women. Am J Clin Nutr 1998;68(Suppl 6):1375S.

4. Jenkins DJ, Kendall CW, Jackson CJ, et al. Effects of high- and low-isoflavone soyfoods on blood lipids, oxidized LDL, homocysteine, and blood pressure in hyperlipidemic men and women. Am J Clin Nutr 2002;76:365.
5. Espeland MA, Hogan PE, Fineberg SE, et al. Effect of postmenopausal hormone therapy on glucose and insulin concentrations. Diabetes Care 1998;21:1589.

6. Thomas BF, Zeisel SH, Busby MG, et al. Quantitative analysis of the principal soy isoflavones genistein, daidzein and glycitein, and their primary conjugated metabolites in human plasma and urine using reversed-phase high-performance liquid chromatography with ultraviolet detection. J Chromatogr B Biomed Sci Appl 2001;760:191.

7. Writing Group for the Women's Health Initiative Investigators. Risks and benefits of estrogen plus progestin in healthy postmenopaual women. JAMA 2002;288:321.

8. Anderson JW, Johnstone BM, Cook-Newell ME. Meta-analysis of the effects of soy protein intake on serum lipids. N Engl J Med 1995;333:276.

9. Dent SB, Peterson CT, Brace LD, et al. Soy protein intake by perimenopausal women does not affect circulating lipids and lipoproteins or coagulation and fibrinolytic factors. J Nutr 2001;131:2280.

10. Crouse JR 3rd, Morgan T, Terry JG, Ellis J, Vitolins M, Burke GL. A randomized trial comparing the effect of casein with that of soy protein containing varying amounts of isoflavones on plasma concentrations of lipids and lipoproteins. Arch Intern Med 1999;159:2070.

11. Hodgson JM, Puddey IB, Beilin LJ, Mori TA, Croft KD. Supplementation with isoflavonoid phytoestrogens does not alter serum lipid concentrations: A randomized controlled trial in humans. J Nutr 1998; 128:728.

12. Nestel PJ, Pomeroy S, Kay S, et al. Isoflavone from red clover improves systemic arterial compliance but not plasma lipids in menopausal women. J Clin Endocrinol Metab 1999;84:895.

13. Nestel PJ, Yamashita $T$, Sasahara $T$, et al. Soy isoflavones improve systemic arterial compliance but not plasma lipids in menopausal and perimenopausal women. Arterioscler Thromb Vasc Biol 1997;17:3392.

14. Simons LA, von Konigsmark M, Simons J, Celermajer D. Phytoestrogens do not influence lipoprotein levels or endothelial function in healthy, postmenopausal women. Am J Cardiol 2000;85:1297.

15. Vedavanam K, Srijayanta S, O'Reilly J, Raman A, Wiseman $H$. Antioxidant action and potential antidiabetic properties of an isoflavonoid-containing soyabean phytochemical extract (SPE). Phytother Res 1999;13:601.

16. Lee DS, Lee SH. Genistein, a soy isoflavone, is a potent alpha-glucosidase inhibitor. FEBS Lett 2001; 501:84.

17. Sorenson RL, Brelje TC, Roth C. Effect of tyrosine kinase inhibitors on islets of Langerhans: Evidence for tyrosine kinases in the regulation of insulin secretion. Endocrinology 1994;134:1975.

18. Dang ZC, Audinot V, Papapoulos SE, Boutin JA, Lowik CW. Peroxisome proliferator-activated receptor gamma (PPARgamma) as a molecular target for the soy phytoestrogen genistein. J Biol Chem 2003; 278:962. 
19. Mezei O, Banz WJ, Steger RW, Peluso MR, Winters TA, Shay N. Soy isoflavones exert antidiabetic and hypolipidemic effects through the PPAR pathways in obese Zucker rats and murine RAW 264.7 cells. J Nutr 2003;133:1238.

20. Bhathena SJ, Velasquez MT. Beneficial role of dietary phytoestrogens in obesity and diabetes. Am J Clin Nutr 2002;76:1191.

21. Fineberg SE. Glycaemic control and hormone replacement therapy: Implications of the Postmenopausal Estrogen/Progestogen Intervention (PEPI) study. Drugs Aging 2000;17:453.

22. Jayagopal V, Albertazzi P, Kilpatrick ES, et al. Beneficial effects of soy phytoestrogen intake in postmenopausal women with type 2 diabetes. Diabetes Care 2002;25:1709.
23. Goodman-Gruen D, Kritz-Silverstein D. Usual dietary isoflavone intake is associated with cardiovascular disease risk factor in postmenopausal women. J Nutr 2001;131:1201.

Address reprint requests to: Shao-Yi Cheng, M.D., M.P.H. Department of Family Medicine National Taiwan University Hospital 7, Chung-Shan South Road 10016 Taipei Taiwan

E-mail: cheng214@ha.mc.ntu.edu.tw 